\title{
AN EFFICIENT FEATURE EXTRACTION METHOD WITH PSEUDO-ZERNIKE MOMENT FOR FACIAL RECOGNITION OF IDENTICAL TWINS
}

\author{
Hoda Marouf ${ }^{1}$ and Karim Faez ${ }^{2}$ \\ ${ }^{1}$ Department of Electrical, Computer and Biomedical Engineering, Qazvin branch, \\ Islamic Azad University, Qazvin, Iran \\ ${ }^{2}$ Department of Electrical Engineering, Amirkabir University of Technology, Tehran, \\ Iran
}

\begin{abstract}
Face recognition is one of the most challenging problems in the domain of image processing and machine vision. Face recognition system is critical when individuals have very similar biometric signature such as identical twins. In this paper, new efficient facial-based identical twins recognition is proposed according to the geometric moment. The utilized geometric moment is Pseudo-Zernike Moment (PZM) as a feature extractor inside the facial area of identical twins images. Also, the facial area inside an image is detected using Ada Boost approach. The proposed method is evaluated on two datasets, Twins Days Festival and Iranian Twin Society which contain scaled, which contain the shifted and rotated facial images of identical twins in different illuminations. The results prove the ability of proposed method to recognize a pair of identical twins. Also, results show that the proposed method is robust to rotation, scaling and changing illumination.
\end{abstract}

\section{KEYWORDS}

Face Recognition, Identical Twins, Invariant Moment, Pseudo-Zernike Moment

\section{INTRODUCTION}

Human face is considered as a suitable property to identify people from his (her) image. Along with this property, recognition of facial of identical twin is one of the most challenging problems in pattern recognition applications because of the similarity between a pair of twin. In the domain of facial identical twins recognition, previous works are listed as: in [12], Klare and Jain introduced a face detection algorithm which includes three levels. In the first level, overall appearance of the face is constructed; in the second level, exact geometric and structural embedment of face with differentiating between two similar faces are performed; and finally, the third level consists of process of skin disorders detection such as wounds, and so on. Sun et al. [16] utilized Cognitec FaceVACS system to recognize identical twins from CASIA Multimodal Biometrics Database. They obtained true accept rate of approximately90\% at a false accept rate greater than 10\%. Park et al. [14] proposed an identical twins recognition algorithm that consists of three steps: in the first step, the proposed method marks the face images using normal geometric methods; in the second step, the Euclidean distance between a pair of markers, of test image and images in dataset, are measured and compared; and the final step involves nding the strong similarity on the marked regions. Srinivas et al. [15] studied on distinguishing of twins using marks on the face image. Martinet al. [3] employed DNA approach to recognize identical

DOI : 10.5121/ijcsity.2014.2101 
International Journal of Computational Science and Information Technology (IJCSITY) Vol.2, No.1, February 2014 twins. In this paper, we use the geometric moments to extract feature vector from facial images of twins to recognize identical twins.

This paper is organized as follow: feature extraction step of a face recognition system is introduced in Section 2. The proposed method is presented in Section 3. Experimental results are described in Section 4 and the paper will be concluded in Section 5.

\section{FEATURE EXTRACTION}

Each face detection system contains four steps: preprocessing, face localization, feature extraction and classification. Feature extraction refers to the extraction of useful information from raw data so that they are suitable for the classification process. The feature extraction stage is characterized by a series of input patterns. The major problem of feature extraction is that it depends on application and the feature extraction methods are not public. Feature extraction methods can be divided into two majors: structural features and statistical features [11][19]. The first group is based on local structure of image. It means that the structural features deals with local data. Facial change or change in environmental conditions is the major problem of the structural features [7].

In the statistics-based feature extraction techniques, global data is employed to create a set of feature vector elements in order to perform recognition. A mixture of irrelevant data, which are usually part of a facial image, may result in an incorrect set of feature vector elements. Therefore, data that are irrelevant to facial portion such as hair, shoulders, and background should be regarded in the feature extraction phase [10]. The statistics-based feature extraction techniques are Principle Component Analysis (PCA), Legendre Moment(LM) [13] and Zernike Moments (ZM) [20], Pseudo-Zernike Moment (PZM) [8]. Legendre functions are Legendre differential equation. The main advantage is that Legendre moments like Legendre basis functions are orthogonal. Legendre moments are independent of each other and are free of data redundancy.

Zernike Moment (ZM) is a set of orthogonal polynomials on the unit disk. The ZM technique is independent of scale and rotation of face in image. The Pseudo-Zernike Moment (PZM) is the same as ZM but number of features in PZM is more than the ZM and so, PZM is more appropriate than $\mathrm{ZM}$ for recognition of identical twins.In this study, we use PZM to extract feature elements in order to recognize identical twins. The PZM will be described in the next Section.

\section{Proposed Method}

The main goal of this paper is to distinguish the identical twins using face recognition. For this purpose, AdaBoost [18] technique is used for face localization and subimage creation.After that the PZM technique is employed to extract feature vector from the subimage oftest image. After that the feature vectors inside the subimages of all images in dataset are obtained using PZM approach. Finally, comparison between the feature vector of test image and the feature vectors of all images of dataset is done to select the closest image from dataset as the pair of test image. In the next Section, the AdaBoost face detection, PZM and its task of feature vector creation are described.

\subsection{Face Detection Method}

As the mentioned before, face detection step is the second step of this algorithm to recognize identical twins. This step is based on the combing of successively more complex classifiersin a 
International Journal of Computational Science and Information Technology (IJCSITY) Vol.2, No.1, February 2014 cascade structure using AdaBoost [18]. Furthermore, the AdaBoost technique is used to select a small number of Haar-like features [18].

After finding an object in an image as a face candidate, an ellipse is drowning around the main location of face in an image[8]. For this purpose, an ellipse model is constructed using five parameters: $X_{0}$ and $Y_{0}$ are the centers of the ellipse, $\theta$ is the orientation, $\alpha$ and $\beta$ are the minor and the major axes of the ellipse, respectively. Before the calculation of these parameters, geometric moments are required to describe. The geometric moments of order $p+q$ of a digital image are defined as

$$
M_{p q}=\Sigma_{x} \Sigma_{y} f(x, y) x^{p} y^{Q}(1)
$$

Where $p, q=0,1,2, \ldots$ and $f(x, y)$ is the grey-scale value of the digital image at $x$ and $y$ location. The translation invariant central moments are obtained by placing origin at thecenter of the image:

$\mu_{p q}=\Sigma_{x} \Sigma_{y} f(x, y)\left(x-x_{0}\right)^{p}\left(y-y_{0}\right)^{q}(2)$

Where $x_{0}=\frac{M_{10}}{M_{00}}$ and $x_{0}=\frac{M_{01}}{M_{00}}$ yare the centers of the connected components. Thus, center of gravity of the connected components is used as the center of the ellipse. The orientation of the ellipse is computed by determining the least moment of inertia [8].

$\theta=\frac{1}{2} \arctan \left(\frac{2 \mu_{11}}{\mu_{s 0}-\mu_{02}}\right)(3)$

Where $\mu_{p q}$ shows the central moment of the connected components as described in (2). The length of the major and the minor axes of the best-fit ellipse can also be computed by evaluating the moment of inertia. With the least and the greatest moments of inertia of an ellipse defined as

$I_{\min }=\Sigma_{x} \Sigma_{y}\left[\left(x-x_{0}\right) \cos \theta-\left(y-y_{0}\right) \sin \theta\right]^{2}(4)$

$l_{\max }=\Sigma_{x} \Sigma_{y}\left[\left(x-x_{0}\right) \sin \theta-\left(y-y_{0}\right) \cos \theta\right]^{2}(5)$

Length of the major and the minor axes are calculated from [8] as

$\alpha=\frac{1}{\pi\left[I_{\text {max }}^{s} / I_{\min }\right]^{-\frac{1}{8}}}(6)$
$\beta=\frac{1}{\pi\left[I_{\min }^{8} / I_{\max }\right]^{\frac{1}{8}}}(7)$

To determine how well the best-fit ellipse approximates the connected components, a distance measure between the connected components and the best-fit ellipse is calculated as follows [8]

$\emptyset_{i}=\frac{P_{\text {irside }}}{\text { linn }}$

$\emptyset_{0}=\frac{p_{\text {cutside }}^{\text {inn }}}{\mu_{00}}(9)$

where the $P_{\text {inside }}$ is the number of background points inside the ellipse, $P_{\text {outside }}$ is the number of points of the connected components that are outside the ellipse, and $\mu_{00}$ is the size of the connected components. After drawing of ellipse, a subimage is made according to the ellipse and finally, the PZM is used to extract features inside the subimage. 
International Journal of Computational Science and Information Technology (IJCSITY) Vol.2, No.1, February 2014

\subsection{Zernike Moment (ZM)}

PZM is geometric-based moment that uses the global information in an image for extracting features [9]. The orthogonal moments of PZM are shift, rotation, and scale invariants which are suitable for pattern recognition applications [6][5][8][17]. Pseudo-Zernike contains several orthogonal sets of complex-valued polynomials defined as

$$
V_{n m}(x, y)=R_{n m}(x, y) \exp \left(j m \tan ^{-1}\left(\frac{y}{x}\right)\right)
$$

where $x^{2}+y^{2} \leq 1, n \geq 0,|m| \leq n$, and the radial polynomials $\left\{R_{n m}\right\}$ are defined as

$$
R_{n m}(x, y)=\sum_{s=0}^{(n-|m|)} D_{n .|m|, s}\left(x^{2}+y^{2}\right)^{\frac{n-4}{2}}
$$

where

$$
D_{n,|m|, s}=(-1)^{s} \frac{(2 n+1-s) !}{a !(n-|m|-o) !(n-|m|-o+1) !}
$$

The ZM of order $n$ and repetition $m$ can be computed as

$$
P Z M_{n m}=\frac{n+1}{\pi} \sum_{x} \Sigma_{y} f(x, y) V_{n m}^{*}(x, y)
$$

It should be noted that the PZM is computed for positive $m$ because $V_{n m}(x, y)=V_{n m}^{*}(x, y)$.If an image is rotated, phase of moments in PZM will be varied and its absolute value remains constant [4]. Thus, if the absolute value or value of PZM is considered as the feature,the feature $f$ is independent of rotation. In the next Section, we will focus on creating of feature vector using PZM.

\subsection{Creating feature vector}

After face localization and subimage creation, the PZM is computed for each subimage as the face features. The feature vector elements are defined according to PZM orders as follows:

$F V_{j}=\left\{P Z M_{k m} \mid k=j, j+1, \ldots, N\right\}$

where $j$ is interval $[1, N-1]$ and so, $F V_{j}$ contains all the PZM from order $j$ to $N$. Samples of feature vector elements will be demonstrated in Table 1 for $j=3,5$ and 9, and $N=10$. As Table1 shows, increasing of $j$ decreases the number of elements in each feature vector $\left(F V_{j}\right)$.

\section{EXPERIMENTAL RESULTS}

The proposed method is evaluated on two datasets: Twins Days Festival [2] and Iranian Twin Society [1] which contain 520 and 600 pairs of identical twins images, respectively. The used datasets contain the scaled and rotated faces in different illuminations. Figure 1shows the subimages of some twin test images. The results of identical twins recognition of LM [13] is compared with ZM [20] and PZM. Experiments have been carried out in three steps according to order of moment. In the first step, order $n$ is in interval [1,6], in the second step, order $n$ is in interval $[6,8]$ and for third step, order $\mathrm{n}$ is in interval $[9,10](2)$.

In this paper, $\mathrm{N}$ is set $10(\mathrm{~N}=10)$ and $\mathrm{j}$ varies from 1 to 9 . The misclassification rates of all geometric moments (LM, ZM and PZM) are presented in Table 3. The misclassification rate reported in the table are computed as 
International Journal of Computational Science and Information Technology (IJCSITY) Vol.2, No.1, February 2014

$$
\text { Error rate }=\frac{\text { No. of miscl ssification }}{\text { No. of total testing patterns }}
$$

Table 1. Feature vector elements based on the PZM

\begin{tabular}{|c|c|c|c|}
\hline \multirow{2}{*}{$j$ value } & \multicolumn{2}{|c|}{$\mathrm{FV} \quad$ feature elements $\left(P Z M_{k m}\right)$} & \multirow{2}{*}{$\begin{array}{l}\text { Number of } \\
\text { feature element }\end{array}$} \\
\hline & $\mathbf{K}$ & $\mathbf{M}$ & \\
\hline \multirow{7}{*}{4} & 4 & $0,1,2,3,4$ & \multirow{7}{*}{56} \\
\hline & 5 & $0,1,2,3,4,5$ & \\
\hline & 6 & $0,1,2,3,4,5,6$ & \\
\hline & 7 & $0,1,2,3,4,5,6,7$ & \\
\hline & 8 & $0,1,2,3,4,5,6,7,8$ & \\
\hline & 9 & $0,1,2,3,4,5,6,7,8,9$ & \\
\hline & 10 & $0,1,2,3,4,5,6,7,8,9,10$ & \\
\hline \multirow{5}{*}{6} & 6 & $0,1,2,3,4,5,6$ & \multirow{5}{*}{45} \\
\hline & 7 & $0,1,2,3,4,5,6,7$ & \\
\hline & 8 & $0,1,2,3,4,5,6,7,8$ & \\
\hline & 9 & $0,1,2,3,4,5,6,7,8,9$ & \\
\hline & 10 & $0,1,2,3,4,5,6,7,8,9,10$ & \\
\hline \multirow{2}{*}{9} & 9 & $0,1,2,3,4,5,6,7,8,9$ & \multirow{2}{*}{21} \\
\hline & 10 & $0,1,2,3,4,5,6,7,8,9,10$ & \\
\hline
\end{tabular}

Table 2. Feature vector elements produced by geometric moments in each experiment.

\begin{tabular}{||c|l|l|l||}
\hline Cat. & LM feature elements & ZM feature elements & PZM feature elements \\
& $n=1, m=1$ & $n=1, m=1$ & $n=1, m=0,1$ \\
& $n=2, m=0,2$ & $n=2, m=0,2$ & $n=2, m=0,1,2$ \\
1 & $n=3, m=1,3$ & $n=3, m=1,3$ & $n=3, m=0,1,2,3$ \\
& $n=4, m=0,2,4$ & $n=4, m=0,2,4$ & $n=4, m=0,1,2,3,4$ \\
& $n=5, m=1,3,5$ & $n=5, m=1,3,5$ & $n=5, m=0,1,2,3,4,5$ \\
& $n=6, m=0,2,4,6$ & $n=6, m=0,2,4,6$ & $n=6, m=0,1,2,3,4,5,6$ \\
\hline \multirow{3}{*}{2} & $n=6, m=0,2,4,6$ & $n=6, m=0,2,4,6$ & $n=6, m=0,1,2,3,4,5,6$ \\
& $n=7, m=1,3,5,7$ & $n=7, m=1,3,5,7$ & $n=7, m=0,1,2,3,4,5,6,7$ \\
& $n=8, m=0,2,4,6,8$ & $n=8, m=0,2,4,6,8$ & $n=8, m=0,1,2,3,4,5,6,7,8$ \\
\hline \multirow{2}{*}{3} & $n=9, m=1,3,5,7,9$ & $n=9, m=1,3,5,7,9$ & $n=9, m=0,1,2,3,4,5,6,7,8,9$ \\
& $n=10, m=0,2,4,6,8,10$ & $n=10, m=, 0,2,4,6,8,10$ & $n=10, m=, 0,1,2,3,4,5,6,7,8,9,10$ \\
\hline
\end{tabular}

Table 3 shows misclassification rates of LM, ZM and PZM. Comparison between the geometric moments in Table 3 proves that higher order moments of the PZM have most information for face recognition while low-order moments have no significant effect on the system error. According to the Table, LM achieves high misclassification rate on recognition of twins because the rotation of face in an image has bad effect on the performance of LM. As the Table 3 shows, the misclassification rate of PZM is lower than the ZM because PZM is shift, rotation and scale invariant while the $\mathrm{ZM}$ is shift and scale invariant. Furthermore, number of feature elements produced by PZM is more than the ZM and so the global information captured by PZM is sufficient for recognition of twins. Visual results of PZM on pair of identical twins are illustrated in Figure 2 which refers to the Twins Days Festival [2] and Iranian Twin Society [1] datasets, respectively. The first row of the Table refers to the results of PZM on the Twins Days Festival dataset[2] and the second row is the results of PZM on the Iranian Twin Society dataset[1]. 
International Journal of Computational Science and Information Technology (IJCSITY) Vol.2, No.1, February 2014

Table 3. Error rate of each geometric moment in different categories. Following parameters are means as No (Number), FE (Feature Elements), Mis. (Misclassification) and ER (Error Rate).

\begin{tabular}{|c|c|c|c|c|c|c|c|c|c|}
\hline \multirow[b]{2}{*}{ Cat. } & \multicolumn{3}{|c|}{ LM } & \multicolumn{3}{|c|}{ ZM } & \multicolumn{3}{|c|}{ PZM } \\
\hline & $\begin{array}{l}\text { No. of } \\
\text { FE }\end{array}$ & $\begin{array}{l}\text { No. of } \\
\text { Mis. }\end{array}$ & ER & $\begin{array}{l}\text { No. of } \\
\text { FE }\end{array}$ & $\begin{array}{l}\text { No. of } \\
\text { Mis. }\end{array}$ & ER & $\begin{array}{l}\text { No. of } \\
\text { FE }\end{array}$ & $\begin{array}{l}\text { No. of } \\
\text { Mis. }\end{array}$ & ER \\
\hline $\mathrm{n}=1,2, \ldots, 6$ & 15 & 20 & $10 \%$ & 15 & 17 & $8.5 \%$ & 26 & 9 & $4.5 \%$ \\
\hline $\mathrm{n}=6,7,8$ & 13 & 18 & $9.1 \%$ & 13 & 13 & $6.5 \%$ & 24 & 6 & $3 \%$ \\
\hline $\mathrm{n}=9,10$ & 11 & 12 & $6.1 \%$ & 11 & 8 & $4 \%$ & 21 & 3 & $1.3 \%$ \\
\hline
\end{tabular}
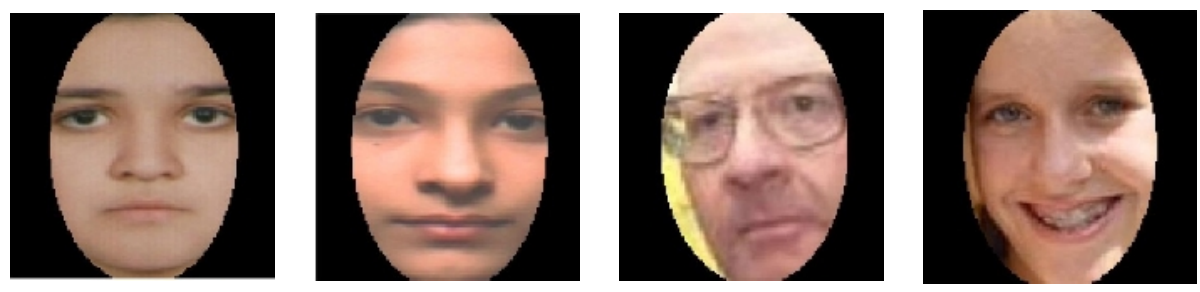

Figure 1. Creating of subimage based on the ellipse formation.
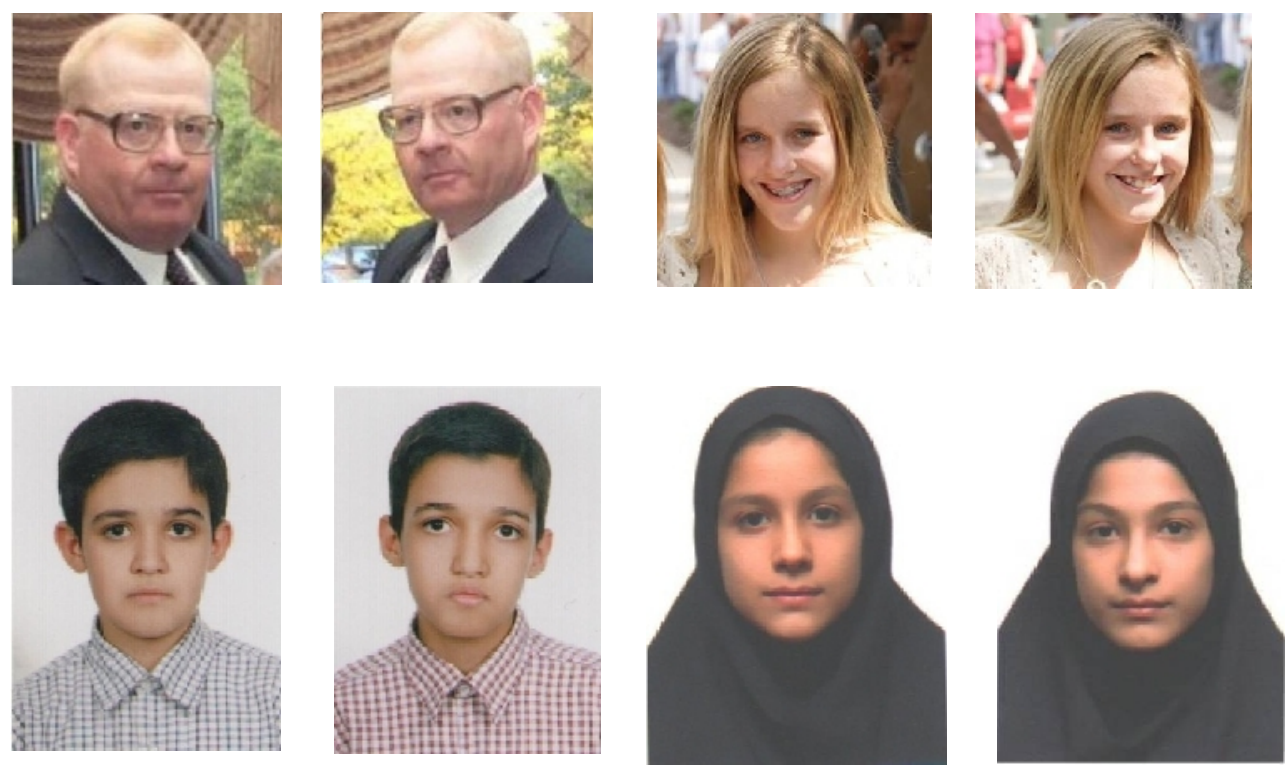

Figure 2. Samples of testing identical twins which were correctly classified by PZM. The firstrow refers to the results of PZM on the Twins Days Festival dataset [2] and the second row is the results of PZM on the Iranian Twin Society dataset [1].

According to the numerical and visual results, PZM is able to create informative feature vector inside the subimages of a pair of identical twins which is necessary for recognition of identical twins. The results prove that PZM is scale, shift and rotation invariant. 


\section{Conclusions}

This paper is focused on the improving of face recognition systems for distinguishing of a pair of identical twins. The proposed method is based on the Pseudo-Zernike Moment (PZM) as a feature extractor to recognize a pair of identical twins. Also, the location of face in an image is detected using the AdaBoost method and then the PZM is utilized to construct feature vector elements. Experimental results on two datasets show that the proposed method is superior to the other geometric moments such as Zernike Moment (ZM) and Legendre Moment (LM)and also is robust to rotation and scaling and changing illumination.

\section{REFERENCES}

[1] Iranian twin society. http://www.irtwins.com/.

[2] Twins days festival. http://www.twinsdays.org.

[3] Arias, E., MacDorman, M.F., Strobino, D.M.\&Guyer, B. (2003)“Annual summary of vitalstatistics2002”,Pediatrics,Vol. 112, No. 6, pp. 1215-1230.

[4] Bailey, R.R. (1993)“Automatic recognition of handwritten numerals via orthogonal momentsusing statistical and neural network classifiers”, Ph.D. thesis, Dallas, TX, USA (1993),aAI9331109.

[5] Belhumeur, P., Hespanha, J.\&Kriegman, D. (1997)“Eigenfaces vs. fisherfaces recognition using classspecific linear projection”, IEEETransactions on Pattern Analysis and Machine Intelligence,Vol. 19, No. 7, pp. 711-720.

[6] Belkasim, S., Shridhar, M.\&Ahmadi, M. (1991)"Pattern recognition with moment invariants:A comparative study and new results",Pattern Recognition,Vol. 24, No.12, pp. 1117 1138,http://www.sciencedirect.com/science/article/pii/003132039190140Z.

[7] Bichsel, M.\&Pentland, A. (1994)“Human face recognition and the face image sets topology" CVGIP Image Understanding,Vol. 59, No. 2, pp. 254-261.

[8] Haddadnia, J., Ahmadi, M.\&Faez, K. (2002)“An efficient method for recognition of human facesusing higher orders pseudo Zernike moment invariant”,In: Proceedings. Fifth IEEE International Conference on Automatic Face and Gesture Recognition 2002, pp. 330-335.

[9] Haddadnia, J., Faez, K.\&Moallem, P. (2001)"Neural network based face recognition with moment invariants",In Proceedings of Int. Conference on Image Processing 2001, Vol. 1, pp. 1018-1021.

[10] Haddadnia, J., Ahmadi, M.\&Faez, K. (2003)“An efficient feature extraction method withpseudoZernike moment in RBF neural network-based human face recognition system”, EURASIP Journal of Appl. Signal Process,pp. 890-901, http://dx.doi.org/10. 1155/S1110865703305128.

[11] Hjelms, E.\& Low, B.K. (2001)“Face detection: A survey",Computer Vision and Image Understanding, Vol. 83, No. 3, PP. 236-274.

[12] Klare, B.\& Jain, A. (2010)“On a taxonomy of facial features”,In Fourth IEEE International Conference on Biometrics, Theory Applications and Systems (BTAS), pp. 1-8.

[13] Liao, S.\&Pawlak, M. (1996)“On image analysis by moments”,IEEE Transactions on Pattern Analysis and Machine Intelligence, Vol. 18, No. 3, pp. 254-266.

[14] Park, U., Jillela, R., Ross, A.\& Jain, A. (2011) "Periocular biometrics in the visible spectrum", IEEE Transactions on Information Forensics and Security, Vol. 6 No. 1, pp. 96-106.

[15] Srinivas, N., Aggarwal, G., Flynn, P.\&VorderBruegge, R. (2012)“Analysis of facial marks todistinguish between identical twins", IEEE Transactions on Information Forensics and Security, Vol. 7, No. 5, pp. 1536-1550.

[16] Sun, Z., Paulino, A.A., Feng, J., Chai, Z., Tan, T.\& Jain, A.K.(2010) “A study of multibiometrictraits of identical twins",http://dx.doi.org/10.1117/12.851369.

[17] Teh, C.H.\& Chin, R.(1988) "On image analysis by the methods of moments". IEEE Transactions on Pattern Analysis and Machine Intelligence, Vol. 10, No. 4, pp. 496-513.

[18] Viola, P.\& Jones, M. (2001)“Rapid object detection using a boosted cascade of simple features", In Proceedings of the IEEE Computer Society Conference on Computer Vision and Pattern Recognition 2001 (CVPR'01),Vol. 1, pp. I-511-I-518.

[19] Yang, M.H., Kriegman, D., Ahuja, N.(2002) “Detecting faces in images: a survey”,IEEE Transactions on Pattern Analysis and Machine Intelligence, Vol. 24, No. 1, pp. 34-58.

[20] Zernike, v.F.(1934),"Beugungstheorie des schneidenver-fahrens und seiner verbesserten form,der phasenkontrastmethode”,Physica,Vol. 1, No. 7, pp. 689-704. 